\title{
Recent progress on the unified theory of polarization and coherence for stochastic electromagnetic fields
}

Wang, Wei; Zhao, Juan; Hu, Xiaoying; Hanson, Steen Grüner; Takeda, Mitsuo

\section{Published in:}

Proceedings of SPIE

Link to article, DOI:

$10.1117 / 12.2306368$

Publication date:

2017

Document Version

Publisher's PDF, also known as Version of record

Link back to DTU Orbit

Citation (APA):

Wang, W., Zhao, J., Hu, X., Hanson, S. G., \& Takeda, M. (2017). Recent progress on the unified theory of polarization and coherence for stochastic electromagnetic fields. In Proceedings of SPIE (Vol. 10612). [1061202 ] SPIE - International Society for Optical Engineering. Proceedings of SPIE - The International Society for Optical Engineering https://doi.org/10.1117/12.2306368

\section{General rights}

Copyright and moral rights for the publications made accessible in the public portal are retained by the authors and/or other copyright owners and it is a condition of accessing publications that users recognise and abide by the legal requirements associated with these rights.

- Users may download and print one copy of any publication from the public portal for the purpose of private study or research.

- You may not further distribute the material or use it for any profit-making activity or commercial gain

- You may freely distribute the URL identifying the publication in the public portal 


\section{Recent progress on the unified theory of polarization and coherence for stochastic electromagnetic fields}

Wei Wang, Juan Zhao, Xiaoying Hu, Steen G. Hanson, Mitsuo Takeda

Wei Wang, Juan Zhao, Xiaoying Hu, Steen G. Hanson, Mitsuo Takeda, "Recent progress on the unified theory of polarization and coherence for stochastic electromagnetic fields," Proc. SPIE 10612, Thirteenth International Conference on Correlation Optics, 1061202 (18 January 2018); doi: $10.1117 / 12.2306368$

SPIE Event: Thirteenth International Conference on Correlation Optics, 2017, Chernivtsi, Ukraine 


\title{
Recent progress on the unified theory of polarization and coherence for stochastic electromagnetic fields
}

\author{
Wei Wang, a,b,* Juan Zhao, ${ }^{a}$ Xiaoying Hu, ${ }^{\text {a,b }}$ Steen G. Hanson, ${ }^{\mathrm{c}}$ and Mitsuo Takeda ${ }^{\mathrm{d}}$ \\ a Institute of Photonics and Quantum Sciences, School of Engineering and Physical Science, \\ Heriot-Watt University, Edinburgh, United Kingdom, EH14 4AS; \\ ${ }^{\mathrm{b}}$ School of Optoelectronic Engineering, Xi' an Technological University, Xi' an 710032, China \\ ${ }^{c}$ DTU Fotonik, Department of Photonics Engineering, \\ Technical University of Denmark, Dk-4000 Roskilde, Denmark \\ ${ }^{\mathrm{d}}$ Center for Optical Research and Education (CORE), Utsunomiya University, 7-1-2 Yoto, \\ Utsunomiya, Tochigi 321-8585, Japan
}

\begin{abstract}
All optical fields undergo random fluctuation and the underlying theory referred to as coherence and polarization of optical fields has played a fundamental role as an important manifestation of the random fluctuations of the electric fields. In this paper, we reviewed our recent theoretical and experimental work on the unified theory of polarization and coherence including coherence tensor wave, degree of coherence tensor, degree of generalized Stokes parameters, and their applications including coherence tensor holography and two-point resolution of polarimetric imaging.
\end{abstract}

Keywords: Coherence, polarization, mutual coherence tensor, polar-interferometry, polarimetric imaging

\section{INTRODUCTION}

The statistical properties of light play an important role in determining the outcome of most optical experiment. It has long been recognized that correlations provides a very convenient method in describing the statistical properties of light. In particular, the cross correlation between the fluctuating fields at different space time points, known as the coherence function, is a quantity of great interest. The basic wave propagation law for mutual coherence discovery by Wolf has laid a foundation to treat many important problems in statistical optics [1-3]. Another important manifestation of the random fluctuations is the phenomenon of partial polarization. So far, the subjects of coherence and polarization have been investigated extensively and the majority of such study has treated these subjects independently of each other. Yet both are manifestations of the same physical phenomenon, namely of correlations between fluctuations in light beams. Coherence arises from correlations between fluctuations at two or more points in space. Polarization, on other hand, is a manifestation of correlation between fluctuating components of the electric field at a single point.

In recent years, a unification of the phenomena of coherence and polarization has attracted new interests and some developments have been made to demonstrate the usefulness of this more comprehensive formulation of correlation effects in stochastic electromagnetic beams. Inspired by the fact the general second-order coherence tensor of a stationary electromagnetic field obey the wave equation, we have introduced several new concepts, principles and applications of such a new wave referred to as coherence tensor wave. In this paper, we reviewed our recent work on the unified theory of polarization and coherence in stochastic electromagnetic beams including coherence tensor wave, degree of coherence tensor, degree of generalized Stokes parameters, and their applications including coherence tensor holography and twopoint resolution of polarimetric imaging.

*w.wang@hw.ac.uk Tel: +44 (0) 131451 3141; Fax: +44 (0) 1314513129

Thirteenth International Conference on Correlation Optics, edited by

Oleg V. Angelsky, Proc. of SPIE Vol. 10612, 1061202 · (c) 2018 SPIE

CCC code: $0277-786 \mathrm{X} / 18 / \$ 18 \cdot$ doi: $10.1117 / 12.2306368$ 


\section{COHERENCE TENSOR WAVE}

In the unified theory of coherence and polarization of light, the mutual coherence tensor provides an elegant description about the correlation and the polarization properties of a stochastic electromagnetic beam on propagation in free space. The mutual coherence tensor can be regarded as tensor wave because it is known to satisfy in free space a couple of the wave equations

$$
\begin{aligned}
& \nabla_{1}^{2} \mathbf{W}\left(\mathbf{r}_{1}, \mathbf{r}_{2}, \tau\right)=\frac{1}{c^{2}} \frac{\partial^{2}}{\partial \tau^{2}} \mathbf{W}\left(\mathbf{r}_{1}, \mathbf{r}_{2}, \tau\right) \\
& \nabla_{2}^{2} \mathbf{W}\left(\mathbf{r}_{1}, \mathbf{r}_{2}, \tau\right)=\frac{1}{c^{2}} \frac{\partial^{2}}{\partial \tau^{2}} \mathbf{W}\left(\mathbf{r}_{1}, \mathbf{r}_{2}, \tau\right)
\end{aligned}
$$

where $\mathbf{W}\left(\mathbf{r}_{1}, \mathbf{r}_{2}, \tau\right)=W_{m n} \hat{m} \hat{n},(m, n=x, y) \quad$ is the mutual coherence tensor with each element $W_{m n}\left(\mathbf{r}_{1}, \mathbf{r}_{2}, \tau\right)=<\tilde{E}_{m}^{*}\left(\mathbf{r}_{1}, t\right) \tilde{E}_{n}\left(\mathbf{r}_{2}, t+\tau\right)>$ and $\hat{m} \hat{n}$ indicating the basis of a tensor, $\nabla_{1}^{2}$ and $\nabla_{2}^{2}$ are the Laplacian operators acting with respect to $\mathbf{r}_{1}$ and $\mathbf{r}_{2}$, respectively.

Just as an electromagnetic field defined as a vectorial field, the electromagnetic waves are typically regarded as a physical entity characterized by a magnitude whose square is proportional to the energy density and a direction indicating the field fluctuation. In Cartesian coordinate system, we can also decompose the electromagnetic vector into orthogonal components with individual amplitude and phase along $\hat{x}, \hat{y}$ and $\hat{z}$. Learning form the history of science on various kinds of vector and tensor quantities in different physical systems, we can also introduce some new magnitude and unit orientation tensor associated with the mutual coherence tensor wave for stochastic electromagnetic beams.

In the $2 \times 2$ mutual coherence tensor, each component $W_{m n}$ is a complex function with its amplitude $\left|W_{m n}\right|$ and phase $\theta_{m n}$. Similar to the magnitude (length) of a vectorial electric wave, the magnitude (amplitude) of a tensor wave is given by its Euclidean norm [4]

$$
A_{C T W}=\sqrt{\mathbf{W}: \mathbf{W}}=\sqrt{\left|W_{x x}\right|^{2}+\left|W_{x y}\right|^{2}+\left|W_{y y}\right|^{2}+\left|W_{y y}\right|^{2}}
$$

where the subscript CTW indicate a coherence tensor wave, and ' $:$ ' is a double contraction operation with a tensor. Similar to the electric fields where the square of amplitude is the energy density of electric field, the square of $A_{C T W}$ is the coherence energy density of the stochastic electric fields.

In the $2^{\text {nd }}$-order coherence theory of scalar wave fields, the complex degree of coherence is defined as the amplitude of scalar coherence wave $\Gamma$ normalized by the square root of the product of intensity at two pinholes. Therefore, we can introduce the complex degree of coherence tensor for the stochastic electric fields

$$
\boldsymbol{\mu}\left(\mathbf{r}_{1}, \mathbf{r}_{2}\right)=\frac{A_{C T W}}{\sqrt{I\left(\mathbf{r}_{1}\right)} \sqrt{I\left(\mathbf{r}_{2}\right)}} e^{j \theta_{m n}} \hat{m} \hat{n}=\frac{\sqrt{\left|W_{x x}\right|^{2}+\left|W_{x y}\right|^{2}+\left|W_{y x}\right|^{2}+\left|W_{y y}\right|^{2}}}{\sqrt{W_{x x}\left(\mathbf{r}_{1} ; \mathbf{r}_{1}\right)+W_{y y}\left(\mathbf{r}_{1} ; \mathbf{r}_{1}\right)} \sqrt{W_{x x}\left(\mathbf{r}_{2} ; \mathbf{r}_{2}\right)+W_{y y}\left(\mathbf{r}_{2} ; \mathbf{r}_{2}\right)}} e^{j \theta_{m n}} \hat{m} \hat{n},
$$

where. After a comparison of our definition with that in Ref. [5], we can easily find that the square of the modulus for Eq. (3) is equal to the quantity introduced by Tervo and his coworkers, which therefore can be understood as the intensity or power of the degree of coherence for the random electric fields.

By using the algebraic operations, a $2^{\text {nd }}$-order tensor expressed in the Cartesian system as $\mathbf{W}=W_{m n} \hat{m} \hat{n}$ can be rewritten by choosing another orthogonal set of basis tensors. That is

$$
\begin{aligned}
\boldsymbol{W} & =\frac{1}{2}\left(W_{x x}+W_{y y}\right)\left[\begin{array}{ll}
1 & 0 \\
0 & 1
\end{array}\right]+\frac{1}{2}\left(W_{x x}-W_{y y}\right)\left[\begin{array}{cc}
1 & 0 \\
0 & -1
\end{array}\right]+\frac{1}{2}\left(W_{x y}+W_{y x}\right)\left[\begin{array}{ll}
0 & 1 \\
1 & 0
\end{array}\right]+\frac{1}{2 i}\left(W_{x y}-W_{y x}\right)\left[\begin{array}{cc}
0 & i \\
-i & 0
\end{array}\right] \\
& =\frac{1}{2}\left(S_{0} \boldsymbol{\sigma}_{0}+S_{1} \boldsymbol{\sigma}_{1}+S_{2} \boldsymbol{\sigma}_{2}+S_{3} \boldsymbol{\sigma}_{3}\right)
\end{aligned}
$$


where $S_{l}\left(\mathbf{r}_{1}, \mathbf{r}_{2}, \tau\right),(l=0,1,2,3)$ is the generalized Stokes parameters and the new orthogonal basis tensors is

$$
\sigma_{0}=\left[\begin{array}{ll}
1 & 0 \\
0 & 1
\end{array}\right], \sigma_{1}=\left[\begin{array}{cc}
1 & 0 \\
0 & -1
\end{array}\right], \sigma_{2}=\left[\begin{array}{ll}
0 & 1 \\
1 & 0
\end{array}\right], \sigma_{3}=\left[\begin{array}{cc}
0 & i \\
-i & 0
\end{array}\right]
$$

known as Pauli's spin matrix. After straight forward algebra, we can find easily that the amplitude of coherence tensor wave can also be expressed as

$$
A_{C T W}=\sqrt{\boldsymbol{W}: \boldsymbol{W}}=\frac{1}{2} \sqrt{\left|S_{0}\right|^{2}+\left|S_{1}\right|^{2}+\left|S_{2}\right|^{2}+\left|S_{3}\right|^{2}} .
$$

So far, we have outlined several new concepts and principles related to the unified theory of polarization and coherence. We turn now to the application of these principles to some specific examples.

\section{FULL FIELD VISUALIZATION OF COHERENCE TENSOR WAVE}

Experiment has been conducted to observe the coherence tensor wave. Figure 1 is a full-field spatial polarization sensitive coherence visualization system [6]. In our experiment, a piece of plastic plate has been placed before the rotating depolarizer to serve as a partially polarization sample. After a fast-spinning rough-surfaced retardation plate depolarizer, we finally get an extended spatially incoherent polarized source. A radial sheering off-line interferometer is illuminated by this source. The light emitted from the point $O\left(x_{o}, y_{o}\right)$ is collimated by lens $\mathrm{L}_{1}$ and is introduced into this interferometric system. The two telescope systems $\left(\mathrm{L}_{2}-\mathrm{L}_{3}\right.$ and $\left.\mathrm{L}_{2}-\mathrm{L}_{4}\right)$ in two arms of the proposed interferometer share the same lens $\mathrm{L}_{2}$, making the system compact. One beam (Arm A) reflected from mirror $\mathrm{M}_{1}$ is serving as a reference field. The other beam (Arm B) with different magnification reflected from a movable mirror $\mathrm{M}_{2}$ is serving as a three-dimensionally displaced field. And let density filter (DF) be chosen to balance the intensities of the two optical fields at the opposite arms of the interferometer with different magnifications $f_{3} / f_{2}$ and $f_{4} / f_{2}$. Two linear polarizers $P_{1}$ and $P_{2}$ are inserted into the two arms to select desired $x$ - or $y$ - polarization components of electric fields. A $45^{\circ}$ linear polarizers $P_{3}$ is placed in the front of the CCD to shift all orthogonal polarization status into same direction. These two vectorial fields correlate with each other at the observation plane. Due to the fact that the lateral displacement is introduced by the radial shearing from the telescope systems with different magnifications, the longitudinal displacement is introduced by changing the optical path difference from the movable mirror and the polarization is introduced by the polarizers, the interference fringes recorded by the CCD image sensor are the results of superposition of the two fields and the distributions of the mutual intensity matrix can be observed from the fringe visibility [7].

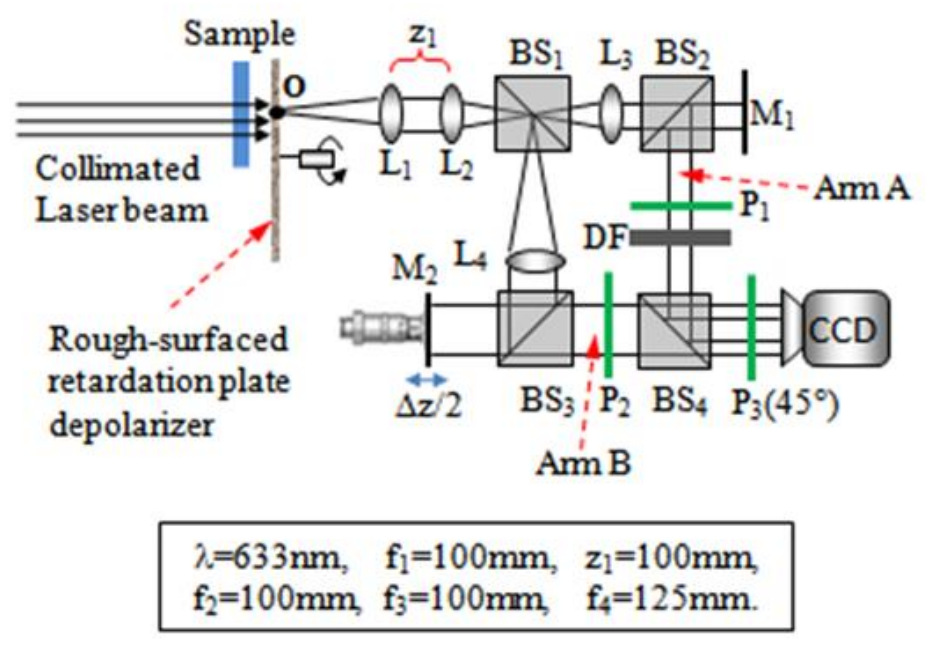

Fig. 1. Full-field polarization sensitive coherence visualization system 


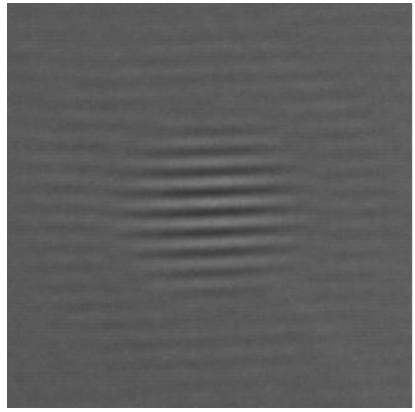

(a)

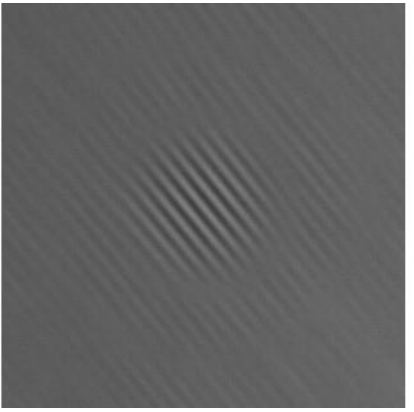

(b)

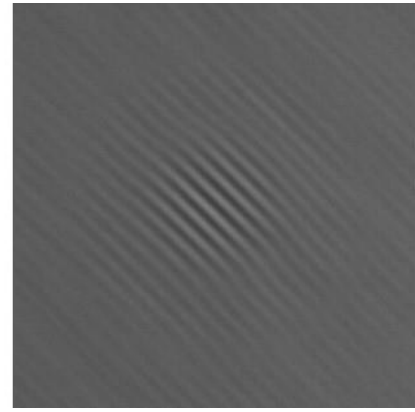

(c)

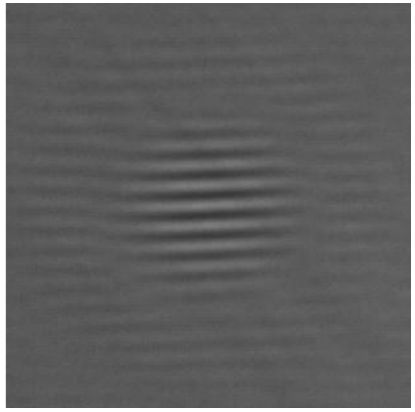

(d)

Figure 2. The recorded interferograms for each elements in mutual coherence matrix. (a): $I_{x x} ;$ (b): $I_{x y} ;$ (c): $I_{y x} ;$ (d): $I_{y y}$.

The experimental results for the recorded interferograms are shown in Figure 2. These images are recorded when the optical path difference of two inference arms equal to zero. By using Fourier fringes analysis [7], we can reconstruct the whole mutual intensity matrix. The distribution of the modulus and the phase for the mutual coherence tensor are shown in Figure 3.

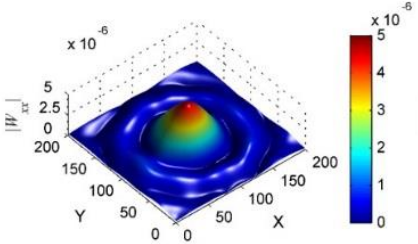

(a)

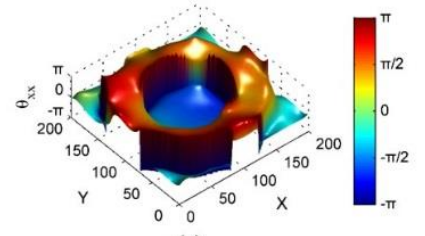

(e)

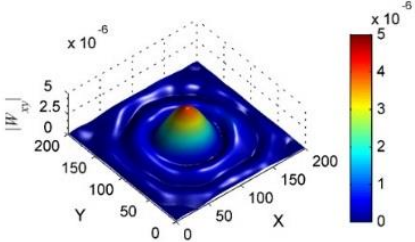

(b)

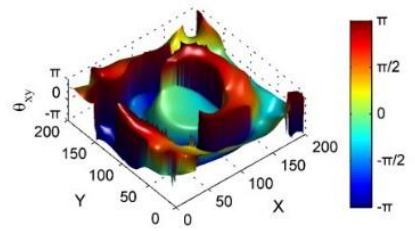

(f)

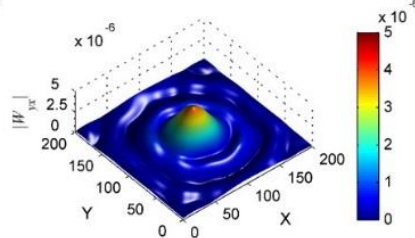

(c)

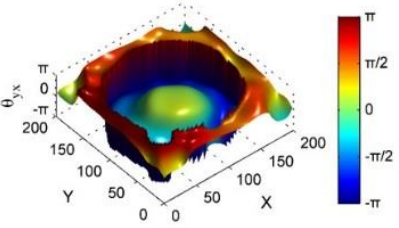

(g)

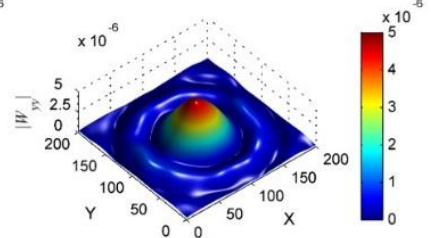

(d)

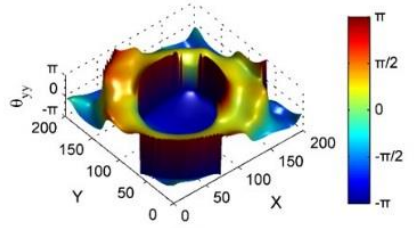

(h)

Figure 3. The modulus and phase of the reconstructed mutual coherence tensor. (a): $\left|\mathbf{W}_{x x}\right| ;$ (b): $\left|\mathbf{W}_{x y}\right| ;$ (c): $\left|\mathbf{W}_{y x}\right| ;(\mathrm{d}):\left|\mathbf{W}_{y y}\right|$;

$$
\text { (e): } \theta_{x x} ;(\mathrm{f}): \theta_{x y} ;(\mathrm{g}): \theta_{y x} ;(\mathrm{h}): \theta_{y y} \text {. }
$$

From the reconstructed mutual coherence tensor, the amplitude of the coherence tensor wave can be obtained through Eq. (2), as shown in Figure 4. Similar to the Airy disk produced by light diffracted when passing through a small circular aperture, the amplitude of coherence tensor wave has the central circular region with high value of optical coherence surrounded by concentric rings with less coherence. The optical coherence for this coherence tensor wave takes local maxima and mimina while it decreases away from the center. From the modulus and phase for the reconstructed mutual coherence tensor, we can also obtain the degree of the coherence tensor based on its definition in Eq. (3). 


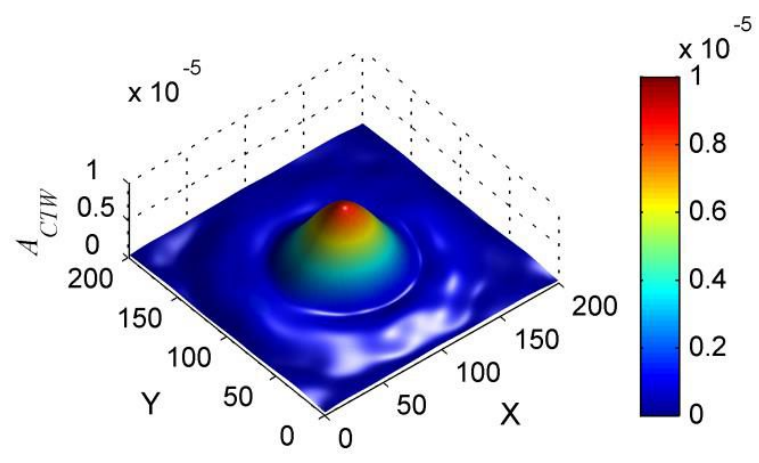

Figure 4. Amplitude of the coherence tensor wave.

\section{COHERENCE TENSOR HOLOGRAPHY}

As one of the application for the unified theory of coherence and polarization, Coherence Tensor Holography (CTH) has been proposed as an direct extension of the coherence holography which is an unconventional holography technique [8]. A computer generated hologram $(\mathrm{CGH})$ with polarization information is illuminated by quasi-monochromatic spatially incoherent light with random polarization and the recorded $3 \mathrm{D}$ object with polarization information is reconstructed by a spatial coherence tensor. The principle of coherence tensor holography is based on the formal analogy between the diffraction integral of polarization interferometry and the formula of the tensor version of van Cittert-Zernike theorem for the stochastic electric fields [9-11].

The experimental setup is shown in Fig. 5 with its optical function divided into two parts. Before the rotating roughsurfaced retardation plate is triangular Sagnac geometry which used to display two orthogonal polarization components [12]. Two computer-generated holograms are firstly loaded by a spatial light modulator (SLM), secondly filtered by a lowpass 4-F system $\left(\mathrm{L}_{2}\right.$ and $\left.\mathrm{L}_{3}\right)$ and finally projected onto the ground glass. The polarizer $\mathrm{P}_{1}$ has a linear polarization at $45^{\circ}$. After the aperture $\mathrm{AP}_{2}$, we have a hologram $\mathrm{H}$ at horizontal polarization and a hologram $\mathrm{V}$ at vertical polarization on the ground glass. After the rotating rough-surfaced retardation plate is a full-field coherence tensor visualization system. Let polarizer $\mathrm{P}_{1}$ and $\mathrm{P}_{2}$ be chosen to transmit only $x$ component and only $y$ component of the incident beam, respectively. A longitudinal motion of the movable mirror $\mathrm{M}_{4}$ produces an axial optical path difference. The polarizer $\mathrm{P}_{4}$, which have a linear polarization at $45^{\circ}$, helps to observe the interference fringes generated from the $x$ component and $y$ component.

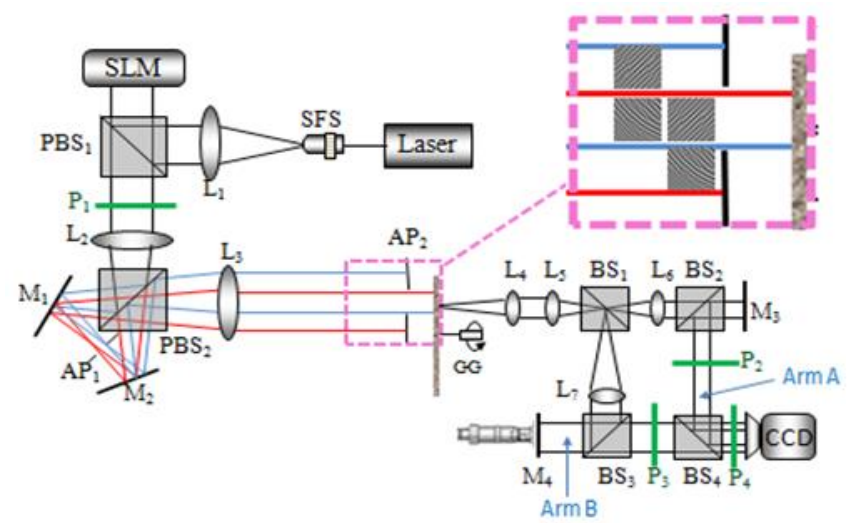

(a)

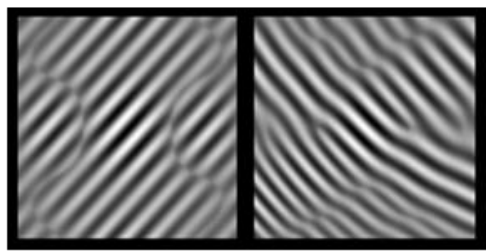

(b)

Fig. 5 Experimental setup for coherence tensor holography (a) and holograms used in experiment, Left one is a hologram generated by a binary object Letter ' $\mathrm{H}$ '; Right one is a hologram generated by a binary object Letter ' $\mathrm{V}$ ' (b). 
The reconstructed coherence images are shown in Fig. 6, where the images at upper row are recorded at coherence focal plane with $Z=0$ and the those at bottom row are recorded at $Z=1 \mathrm{~cm}$. The first column gives the reconstructed Because the hologram $\mathrm{H}$ is an in-focus hologram, its real image can be reconstructed at the location where $Z=0$. Fig. 6(a) has an identical structure to the Letter ' $\mathrm{H}$ ' in the format of the fringe contrast, while Fig. 6(e) has a blurred image of $\mathrm{H}$ for $W_{x x}$ due to the fact of out-of-focus. Fig. 6(d) and (h) are related to the element $W_{y y}$ in the coherence tensor. Similarly, the hologram $\mathrm{V}$ is a out-of-focus hologram for $Z=0$, so its reconstructed image become blurred in Fig. 6(d) and Fig. 6(h) shows a clear image which is identical to the Letter ' $\mathrm{V}$ '. Fig. 6(b) and (f) provide the reconstructed coherence image for $\tilde{W}_{x y}$ and the coherence image for the element $\tilde{W}_{x y}$ in coherence tensor are demonstrated in Fig. 6(c) and (g).

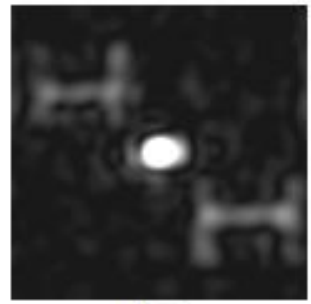

(a)

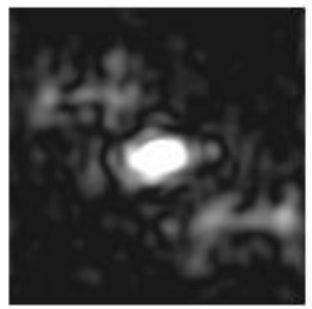

(e)

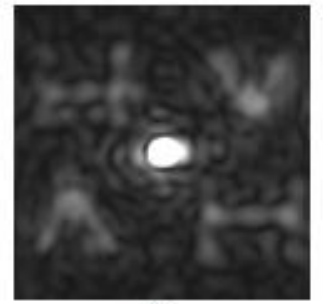

(b)

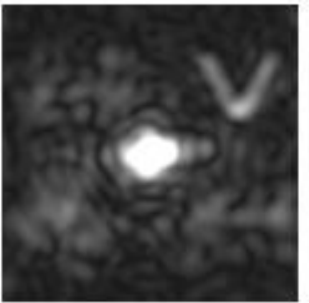

(f)

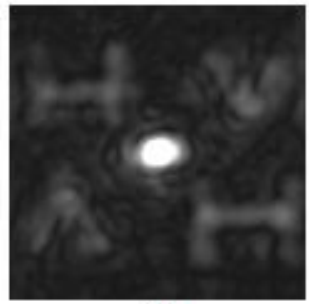

(c)

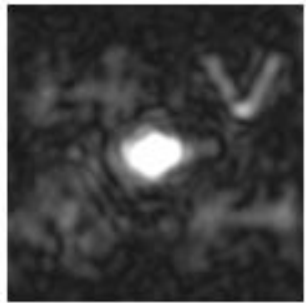

(g)

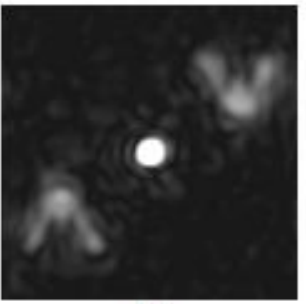

(d)

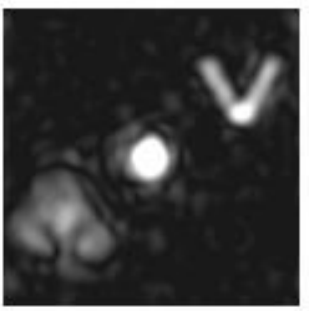

(h)

Fig. 6. Reconstructed coherence images. Top row: (a) the modulus of 4 elements in mutual coherence tensor recorded at coherence focal plane. Bottom row: (b) the modulus of 4 elements in mutual coherence matrix recorded at the location $Z=1 \mathrm{~cm}$.

\section{TWO-POINT RESOLUTION WITH PARTIALLY COHERENT LIGHT FOR POLARIZATION IMAGING}

Optical resolution describes the ability of an imaging system to resolve detail in the object that is being imaged. The twopoint resolution criterion has been long been used as a quality factor for optical systems, particularly in astronomical and bio-medical applications where it has a very real practical significance [13]. It has long been well-known that the partially coherent light has played a very important role in image formation. Although this topic has been studied for more than one hundred years after pioneer work by Lord Rayleigh [14], no investigation has been made to the resolution issue is polarimetric imaging under the partially coherent and partially polarized illumination. Here, we will provide some analysis of the distributions of Stokes parameters for various separations of the two points and various illumination conditions.

Let the object under consideration consists of two equally bright points separated by a distance $2 \Delta$. The generalized Stokes parameters $S_{l}^{o b}\left(\xi_{1}, \eta_{1} ; \xi_{2}, \eta_{2}\right)$ at the object plane is then given by:

$$
S_{l}^{o b}\left(\xi_{1}, \eta_{1} ; \xi_{2}, \eta_{2}\right)=S_{l}^{i n}\left(\xi_{1}, \eta_{1} ; \xi_{2}, \eta_{2}\right)\left[\delta\left(\xi_{1}-\Delta, \eta_{1}\right)+\delta\left(\xi_{1}+\Delta, \eta_{1}\right)\right]\left[\delta\left(\xi_{2}-\Delta, \eta_{2}\right)+\delta\left(\xi_{2}+\Delta, \eta_{2}\right)\right] l=0,1,2,3
$$

where $S_{l}^{i n}\left(\xi_{1}, \eta_{1} ; \xi_{2}, \eta_{2}\right)$ is the generalized Stokes parameters for the incident beam. Then, the Stokes parameters at the imaging plane are given by [15]

$$
S_{l}^{i m}(x, y)=\iiint \int_{-\infty}^{\infty} h\left(x-\xi_{1}, y-\eta_{1}\right) h^{*}\left(x-\xi_{2}, y-\eta_{2}\right) S_{l}^{o b}\left(\xi_{1}, \eta_{1} ; \xi_{2}, \eta_{2}\right) d \xi_{1} d \eta_{1} d \xi_{2} d \eta_{2} \quad l=0,1,2,3,
$$

where $h(x, y ; \xi, \eta)$ is the amplitude impulse response of the imaging system. 
Substituting Eq. (6) into Eq. (7), we have the Stokes parameters for polarimetric imaging

$$
\begin{aligned}
& S_{0}^{\text {im }}(x, 0)=I_{0}\left\{\left[|h(x-\Delta, 0)|^{2}+\left|h^{*}(x+\Delta, 0)\right|^{2}\right]+2 \operatorname{Re}\left[\tilde{\mu}_{S_{0}}(\Delta, 0 ;-\Delta, 0) h(x-\Delta, 0) h^{*}(x+\Delta, 0)\right]\right\}, \\
& S_{1}^{i m}(x, 0)=I_{0}\left\{s_{1}\left[|h(x-\Delta, 0)|^{2}+\left|h^{*}(x+\Delta, 0)\right|^{2}\right]+2 \operatorname{Re}\left[\tilde{\mu}_{S_{1}}(\Delta, 0 ;-\Delta, 0) h(x-\Delta, 0) h^{*}(x+\Delta, 0)\right]\right\}, \\
& S_{2}^{i m}(x, 0)=I_{0}\left\{s_{2}\left[|h(x-\Delta, 0)|^{2}+\left|h^{*}(x+\Delta, 0)\right|^{2}\right]+2 \operatorname{Re}\left[\tilde{\mu}_{S_{2}}(\Delta, 0 ;-\Delta, 0) h(x-\Delta, 0) h^{*}(x+\Delta, 0)\right]\right\}, \\
& S_{3}^{\text {im }}(x, 0)=I_{0}\left\{s_{3}\left[|h(x-\Delta, 0)|^{2}+\left|h^{*}(x+\Delta, 0)\right|^{2}\right]+2 \operatorname{Im}\left[\tilde{\mu}_{S_{3}}(\Delta, 0 ;-\Delta, 0) h(x-\Delta, 0) h^{*}(x+\Delta, 0)\right]\right\},
\end{aligned}
$$

where $s_{l}=S_{l} / S_{0}$ is the normalized Stokes parameters, and $\tilde{\mu}_{S_{l}}\left(\mathbf{r}_{1} ; \mathbf{r}_{2}\right)=S_{l}^{\text {in }}\left(\mathbf{r}_{1} ; \mathbf{r}_{2}\right) /\left(\sqrt{S_{0}\left(\mathbf{r}_{1}\right)} \sqrt{S_{0}\left(\mathbf{r}_{2}\right)}\right)$ is the newly introduced parameters referred to as the degree of the generalized Stokes parameters representing the polarization sensitive correlations at two points. For a diffraction limited imaging system with a circle aperture width of $2 w$, the amplitude impulse response of the equivalent two-dimensional system may be given by

$$
h(x, y)=\frac{2 \pi w^{2}}{(\lambda z)^{2}} \frac{J_{1}\left(2 \pi w \sqrt{x^{2}+y^{2}} / \lambda z\right)}{2 \pi w \sqrt{x^{2}+y^{2}} / \lambda z}
$$

where $J_{1}$ is the Bessel Function of the first kind, $z$ is the image distance, and $\lambda$ is the wavelength. Based on Eqs. (8) and Eq. (9), we are able to estimate the two-point resolution with partially coherent light for polarization imaging.

Some calculation has been made for the distributions of Stokes parameters for various separations of the two points. Fig.6(a) shows the distribution of the Stokes parameter $S_{0}$ plotted as a function of $2 \pi w x / \lambda z$ for a variety of separations of the two points in the object. Here $\delta$ is defined as the dimensionless coordinate $2 \pi w x / \lambda z$, and the degree of the Stokes parameters varies from -1.0 to 1.0 in steps of 0.25 . The position of the ideal image points is indicated in the instance by the broken lines. In each plot, the measured separation of the maxima is not always equivalent to the real point separation. Note the fact that the only measurable quantity is the separation of the two peaks of the resultant distribution for the Stokes parameter $S_{0}$, which corresponds to the real separation of the object points. Hence, we plot in Fig. 6(b) the ratio $R_{S_{0}}$ of the measurable to the ideal separation predicted by geometric optics as a function of the real separation expressed in terms of the dimensionless parameter $\delta$. For a perfect system, clearly, $R_{S_{0}}$ should be unity. The curves plotted in Fig. 2 are for various values of $\tilde{\mu}_{S_{0}}(\Delta, 0 ;-\Delta, 0)$. As shown in Fig. $6(\mathrm{~b})$, when $\tilde{\mu}_{S_{0}}(\Delta, 0 ;-\Delta, 0)=-1.0, R_{S_{0}}$ tends to infinity with a decreasing separation distance indicating that the two points can be always resolved in this case. In addition to this, the other curves are seen to oscillate about the value $R_{S_{0}}=1$ with about a $75 \%$ overshoot in the worst case.

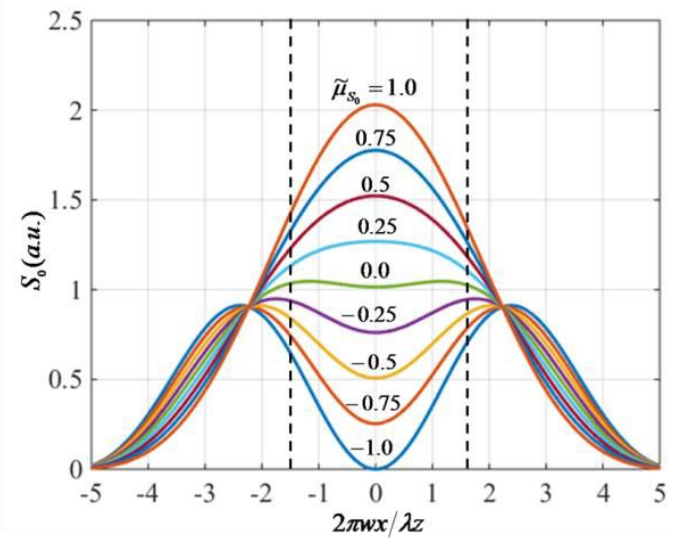

(a)

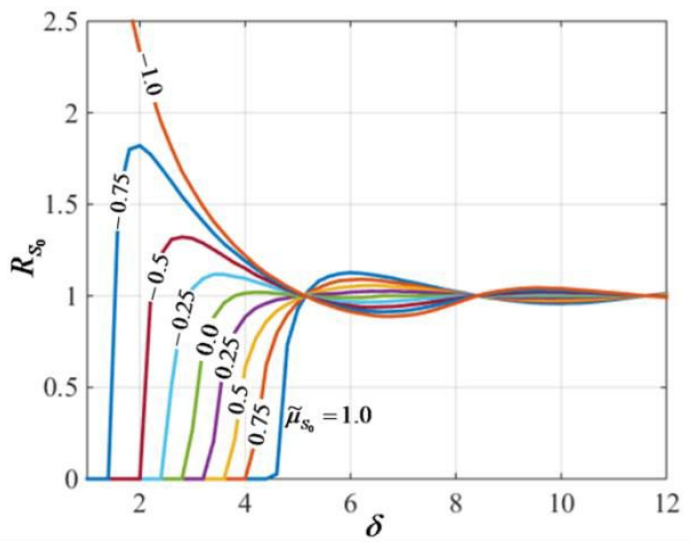

(b)

Fig. 7. Distribution of the Stokes parameters $S_{0}$ for various value of $\mu_{S_{0}}$ (a); The ratio $R_{S_{0}}$ of the measured to the real separation of the two images as a function of the real separation for a diffraction limited imaging system (b).

Figure 8(a) shows the distribution of the Stokes paramet $\mathrm{r}(l=1,2,3)$ plotted as a function of $\delta=2 \pi w x / \lambda z$ for various value of the degree of the general Stoke parameter $\tilde{\mu}_{S_{i}}(\Delta, 0 ;-\Delta, 0)$ and the normalized Stokes parameter $s_{l}$. The ratio $R_{S_{0}}$ of the measurable 
to the ideal separation predicted by geometric optics is given in Fig. 7(b). It's interesting to notice that the two-point resolution for the Stokes parameters in polarimetric imaging will depend on both the degree of the generalized Stokes parameters and the normalized Stokes parameters of the illumination source. When the separation distance is fixed, the two points can be resolvable for certain Stokes parameters and become unresolvable under other Stokes parameter in polarimetric imaging.
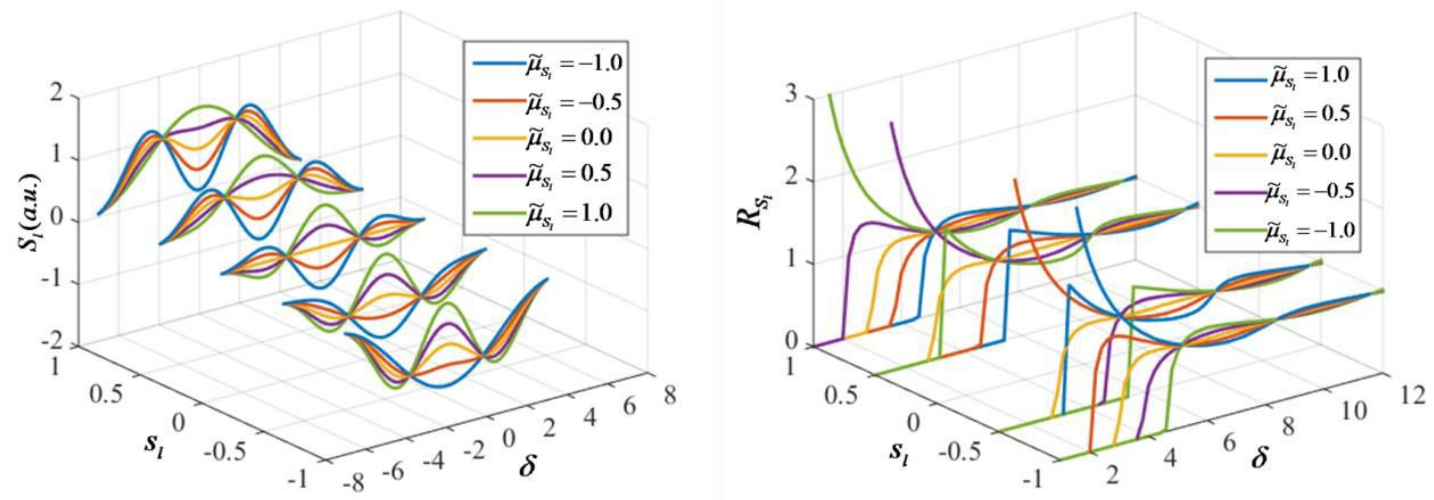

Fig. 9. Distribution of the Stokes parameters $S_{l}(l=1,2,3)$ for various value of $\mu_{S_{0}}$ and $s_{l}$ (a); The ratio $R_{S_{l}}$ of the measured separation to the ideal separation of the two images.

\section{CONCLUSIONS}

In summary, we reviewed our recent work on the unified theory of polarization and coherence in stochastic electromagnetic beams. Based on the wave properties associated with the mutual coherence tensor, we introduced several new concepts associated with the coherence tensor wave and provided the definition for the degree of coherence tensor. We provided a novel optical system for the full-field visualization of coherence tensor and proposed an unconventional holography: coherence tensor holography to synthesize arbitrarily the coherence for stochastic electric fields. The degree of generalized Stokes parameters has been proposed and the two-point resolution issue has been revisited for polarimetric imaging.

\section{REFERENCES}

[1] M. Born, E. Wolf, [Principles of Optics], Cambridge University Press, Chap.10 (1980).

[2] E. Wolf, [Introduction to the Theory of Coherence and Polarization of Light], Cambridge University Press, Cambridge, (2007).

[3] J. W. Goodman, [Statistical Optics], Wiley-interscience, Colorado (2000).

[4] D. E. Neuenschwander, [Tensor Calculus for Physics: A Concise Guide], Johns Hopkins University Press (2014).

[5] J.Tervo. T. Setala, A. T. Friberg, "Degree of coherence for electromagnetic fields," Opt. Express 11, 1137-1143 (2003).

[6] J. Zhao, W. Wang, "Experimental study of spatial coherence diffraction based on full-field coherence visualization," JOSA A31, 2217-2222(2014).

[7] M. Takeda, H. Ina and S. Kobayashi, "Fourier-transform method of fringe-pattern analysis for computer-based topography and interferometry," JOSA A 72, 156-160 (1982).

[8] M. Takeda, W. Wang, Z. Duan, Y. Miyamoto, “Coherence Holography,” Opt. Express 23, 9629-9635 (2005)

[9] T. Shirai, "Some consequences of the van Cittert-Zernike theorem for partially polarized stochastic electromagnetic fields," Opt. Lett. 34, 3761-3763 (2009).

[10] S. Ostrovsky, G. Martinez-Niconoff, Patricia Martinez-Vara, and M. Olvera-Santanaria, "The van Cittert-Zernike theorem for electromagnetic fields," Opt. Express 17, 1746-1752 (2009).

[11] J. Tervo, T. Setala, J. Turunen, and A. Friberg, "Van Cittert-Zernike theorem with Stokes parameters," Opt. Lett. 38, 2301-2303 (2013).

[12] R. K. Singh, D. N. Naik, H. Itou, Y. Miyamoto and M. Takeda, "Vectorial coherence holography," Opt. Express 19, 11558-11567 (2011). 
[13]D. N. Grimes and B. J. Thompson, "Two-Point Resolution with Partially Coherent Light," Journal of the optical society of America, 57, 1330-1334(1967).

[14] Lord Rayleigh, Collected Papers (Cambridge University Press), Cambridge, England, 1902.

[15] O. Korotkova and E. Wolf, "Generalized Stokes parameters of random electromagnetic beams," Opt. Lett. 30, 198-200 (2005). 